\title{
ON BRANCHED COVERS OF THE 2-SPHERE BY THE 2-SPHERE
}

\author{
S. M. GERSTEN
}

(Communicated by Haynes R. Miller)

\begin{abstract}
A new solution of the Hurwitz problem is given for realizing branch data by a branched cover of $S^{2}$ by $S^{2}$. The solution involves products of conjugacy classes in a free group.
\end{abstract}

1. Introduction. A branched cover $f: M \rightarrow N$ of (closed) Riemann surfaces is an open map such that for some finite subset $F$ of $N$ the restriction map $f: M-$ $f^{-1}(F) \rightarrow N-F$ is a covering map of some degree $d>0$, and such that for each point $x \in f^{-1}(F)$ there is a neighborhood $U$ of $x$ in $M$ on which the restricted map $f \mid U: U \rightarrow f(U)$ is equivalent to the map $z \rightarrow z^{e}$ of the complex numbers for some integer $e>0$. The number $e$ is called the ramification number at $x$ and the map $f$ is said to be branched over $y \in N$ if $y \in F$ and if the ramification number at some $x \in f^{-1}(y)$ is at least two.

We may associate to the branched cover $f: M \rightarrow N$ of degree $d$ a branch datum $\mathbf{D}=\mathbf{D}(f)=\left(A_{1}, A_{2}, \ldots, A_{r}\right)$, where $A_{i}=\left[e_{i 1}, e_{i 2}, \ldots, e_{i n_{i}}\right]$ is a partition of $d$. Here the numbers $e_{i}$ are the ramification numbers of the points of the fiber $f^{-1}\left(y_{i}\right)$, where $F=\left\{y_{1}, y_{2}, \ldots, y_{r}\right\}$ is the set of points of $N$ over which $f$ is branched; thus, for each $i$, some $e_{i j} \geq 2$. The datum $\mathrm{D}$ is unique up to order with these conventions. We set $v(\mathbf{D})=\sum_{i, j}\left(e_{i j}-1\right)$. The classical Riemann-Hurwitz formula $[\mathbf{3}, \mathbf{1}]$ states that

$$
\chi(M)=d \chi(N)-v(\mathbf{D}) .
$$

In particular if $M$ and $N$ are both $S^{2}$, then $v(\mathbf{D})=2 d-2$.

The Hurwitz problem asks to what extent condition (1.1) is sufficient for an abstract branch datum $\mathbf{D}=\left(A_{1}, A_{2}, \ldots, A_{r}\right)$ of partitions $A_{i}$ of $d>0$ to be equal to $\mathbf{D}(f)$ for some branched cover $f: M \rightarrow N$ of degree $d$. Hurwitz [3] answered this problem in general. For $N=S^{2}$ his answer is as follows. Given an element $\alpha$ of the symmetric group $S_{d}$ on $d$ symbols, the orbit structure of $\alpha$ determines a partition $\Pi(\alpha)$ of $d$. The abstract branch datum $\mathbf{D}$ as above is realized by a branch cover $f: M \rightarrow S^{2}$ (so $\left.\mathbf{D}=\mathbf{D}(f)\right)$ iff there are permutations $\alpha_{1}, \alpha_{2}, \ldots, \alpha_{r}$ in $S_{d}$ so that

$$
\begin{gathered}
\Pi\left(\alpha_{i}\right)=A_{i}, \quad 1 \leq i \leq r, \\
\alpha_{1} \alpha_{2} \cdots \alpha_{r}=1 \quad \text { in } S_{d},
\end{gathered}
$$

Received by the editors September 8, 1986.

1980 Mathematics Subject Classification (1985 Revision). Primary 57M12.

Key words and phrases. Branched cover, Hurwitz problem, free group, products of conjugacy classes.

Partially supported by NSF Grant DMS 8400882 . 
and

$$
\left\langle\alpha_{1}, \alpha_{2}, \ldots, \alpha_{r}\right\rangle \text { is a transitive subgroup of } S_{d} .
$$

In addition, if (1.2)-(1.4) hold, then $M$ is $S^{2}$ iff $v(\mathbf{D})=2 d-2$, from the RiemannHurwitz formula (1.1). In one sense, then, the Hurwitz problem is solved. Yet there is sufficient difficulty in actually applying Hurwitz criteria in any situation to ask whether more structure exists and whether a more tractible solution can be found using it. For a modern discussion of the Hurwitz problem see [1].

In this article we shall produce a new necessary and sufficient condition that an abstract branch datum $\mathbf{D}$ be realized by a branched cover $f: S^{2} \rightarrow S^{2}$. The result is

(1.5) THEOREM. Let $\mathrm{D}=\left(A_{1}, A_{2}, \ldots, A_{r}\right)$ be an abstract branch datum consisting of partitions $A_{i}$ of $d>0$. Let $A_{i}=\left[e_{i 1}, e_{i 2}, \ldots, e_{i n_{i}}\right], 1 \leq i \leq r$. Let $\Gamma$ be the group presented by $\left\langle x_{1}, x_{2}, \ldots, x_{r} \mid x_{1} x_{2} \cdots x_{r}\right\rangle$. Then $D=\mathbf{D}(f)$ for some branched cover $f: S^{2} \rightarrow S^{2}$ iff both $v(\mathbf{D})=2 d-2$ and

$$
1 \in \prod_{i=1}^{r} \prod_{j=1}^{n_{i}}\left(x_{i}^{e_{i j}}\right)^{\Gamma} .
$$

Here $(g)^{\Gamma}$ denotes the conjugacy class of the element $g \in \Gamma$ and the product in (1.6) is the product, in the group law of $\Gamma$, of subsets of $\Gamma$.

The novelty in (1.6) is the lack of any mention of permutation groups. The answer is a kind of reciprocity law, for it says that the inverse of one factor $x_{i}^{e_{i j}}$ on the right can be expressed as a product of conjugates of the remaining factors.

For example, the datum $\mathbf{D}=([3,3,3],[3,3,3],[2,2,2,2,1])$ satisfies $v(\mathbf{D})=$ $2 d-2, d=9$. But $\mathbf{D}$ is not realizable. For otherwise it would follow from (1.6) that $z \in\left\langle\left\langle x y z, x^{3}, y^{3}, z^{2}\right\rangle\right\rangle \underset{\operatorname{def}}{=} N \subset F(x, y, z)$. If one considers the permutation representation $x \rightarrow(321), y \rightarrow(142), z \rightarrow(13)(24)$ which kills $N$, one sees that this is absurd; the nonrealizability of $\mathbf{D}$ follows.

Here is a second example of the applicability of Theorem (1.5). Let $\mathbf{D}=$ $\{[4,4,1],[3,3,3],[2,2,2,2,1]\}$. Here $v(\mathbf{D})=16$ and $d=9$, so $v(\mathbf{D})=2 d-2$. But $\mathbf{D}$ is not realizable. For otherwise we would have

$$
1 \in\left(x^{4}\right)^{\Gamma} \cdot\left(x^{4}\right)^{\Gamma} \cdot(x)^{\Gamma} \cdot\left(y^{3}\right)^{\Gamma} \cdot\left(y^{3}\right)^{\Gamma} \cdot\left(y^{3}\right)^{\Gamma} \cdot\left(z^{2}\right)^{\Gamma} \cdot\left(z^{2}\right)^{\Gamma} \cdot\left(z^{2}\right)^{\Gamma} \cdot\left(z^{2}\right)^{\Gamma} \cdot(z)^{\Gamma},
$$

where $\Gamma=\langle x, y, z \mid x y z\rangle$. Let $N$ be the normal closure of $x^{4}, y^{3}$ and $z^{2}$ in $\Gamma$. It follows that $x$ and $z^{-1}=x y$ have conjugate images in $\left\langle x, y, z \mid x y z, x^{4}, y^{3}, z^{2}\right\rangle$. But this is absurd as we see by considering the permutation representation $x \rightarrow(4321), z \rightarrow$ (14), $y=x^{-1} z^{-1} \rightarrow(123)$.

A third example will be given in (2.6), where Theorem (1.5) is used to establish the realizability of a branch datum.

I would like to thank the referee for his constructive suggestions in matters of exposition.

2. Constructing branched covers. In this section we assume that $\mathbf{D}=$ $\left(A_{1}, A_{2}, \ldots, A_{r}\right)$ is an abstract branch datum of degree $d \geq 1$, so each $A_{i}$ is a 
partition of $d$. In addition we assume that $v(\mathbf{D})=2 d-2$ and that condition (1.6) is satisfied, so

$$
1 \in \prod_{i=1}^{r} \prod_{j=1}^{n_{i}}\left(x_{i}^{e_{i j}}\right)^{\Gamma},
$$

with $A_{i}=\left[e_{i 1}, e_{i 2}, \ldots, e_{i n_{i}}\right]$ for $1 \leq i \leq r$, where $\Gamma$ is the group of the presentation $\left\langle x_{1}, x_{2}, \ldots, x_{r} \mid x_{1} x_{2} \cdots x_{r}\right\rangle$. Observe that $\Gamma$ is free with free basis $\left\{x_{1}, x_{2}, \ldots, x_{r-1}\right\}$, and $x_{r}=\bar{x}_{r-1} \bar{x}_{r-2} \cdots \bar{x}_{1}$ in $\Gamma$ (we are using the same letters $x_{i}$ for the cosets these elements represent in $\Gamma$ with minimal risk of confusion, and $\bar{x}$ denotes $x^{-1}$ ).

We need to recall the notion of a cancellation diagram introduced in [2]. Let $\Gamma=F\left(x_{1}, x_{2}, \ldots, x_{r-1}\right)$ be the free group freely generated by $x_{1}, x_{2}, \ldots, x_{r-1}$. Let $w_{1}, w_{2}, \ldots, w_{N}$ be any family of nontrivial words in the free monoid generated by $x_{i}$ and $\bar{x}_{i}, 1 \leq i \leq r$. Let $D_{1}, D_{2}, \ldots, D_{N}$ be a family of $N$ pairwise disjoint closed discs in $S^{2}$. Let $Y$ be obtained by removing from $S^{2}$ the interiors of all the discs $D_{i}, 1 \leq i \leq N$. Thus $Y$ is a compact planar surface with $N$ circle boundary components. Let $l_{i}$ be the length of the word $w_{i}$ and mark $l_{i}$ vertices on $\partial D_{i}$ and label these points in order counterclockwise cyclically by the letters of the word $w_{i}$. The reader who wishes a picture can consult $[2,5.1 .1]$.

(2.1) Definition. A cancellation diagram $\mathbf{C}$ for $w_{1}, w_{2}, \ldots, w_{N}[\mathbf{2}, 5.2,5.3]$ is a family of pairwise disjoint arcs, each properly embedded in $Y$, so that each arc begins and ends at vertices of $\partial Y$ labelled by opposite letters $x_{i}, \bar{x}_{i}$ (so e.g. if an arc begins at $x_{j}$, it must end at $\bar{x}_{j}$ ). The reader may consult $[2,5.2 .1]$ for an illustration of a cancellation diagram; a cancellation diagram also appears in (2.6) below.

(2.2) THEOREM. One has $1 \in \prod_{i=1}^{N}\left(w_{i}\right)^{\Gamma}$ iff a cancellation diagram exists for the family $w_{1}, w_{2}, \ldots, w_{N}$.

See $[2,5.2]$ for a proof.

We shall now apply this result to the collection $\mathbf{F}=\left\{x_{i}^{e_{i j}}, 1 \leq i \leq r, 1 \leq j \leq n_{i}\right\}$. Here $x_{r}=\bar{x}_{r-1} \bar{x}_{r-2} \cdots \bar{x}_{1}$, in terms of the free basis $x_{1}, x_{2}, \ldots, x_{r-1}$ for $\Gamma$. The hypothesis (1.6) implies there is a cancellation diagram $\mathbf{C}$ for the family $\mathbf{F}$. Let $Y^{\prime}$ be obtained from $Y$ by cutting $Y$ along the $\operatorname{arcs}$ of $\mathbf{C}$.

Label the closed discs in $S^{2}$ associated with $\mathbf{C}$ by $D_{i j}, 1 \leq i \leq r, 1 \leq j \leq n_{i}$. The boundary of $D_{i j}$ in $Y=S^{2}-\bigcup_{i, j} \stackrel{\circ}{D}_{i j}$ is subdivided by $e_{i j}$ vertices for $1 \leq i \leq r-1$, all labelled $x_{i}$, whereas $D_{r j}$ has $(r-1) \cdot e_{r j}$ vertices on its boundary, with boundary label the cyclic word $\left(\bar{x}_{r-1} \bar{x}_{r-2} \cdots \bar{x}_{1}\right)^{e_{r j}}$.

Let $D_{1}, D_{2}, \ldots, D_{r}$ be pairwise disjoint closed discs in $S^{2}$ and let $X=S^{2}-$ $\bigcup_{i=1}^{r} \stackrel{\circ}{D}_{i}$. We put one vertex on $\partial D_{i}$ labeled $x_{i}$ for $1 \leq i \leq r-1$ and we put $r-1$ vertices on $\partial D_{r}$ labelled in order (counterclockwise) $\bar{x}_{r-1}, \bar{x}_{r-2}, \ldots, \bar{x}_{1}$. There is an obvious cancellation diagram for the collection

$$
\mathbf{F}^{\prime}=\left\{x_{1}, x_{2}, \ldots, x_{r-1}, \bar{x}_{r-1} \bar{x}_{r-2}, \ldots, \bar{x}_{1}\right\}
$$

obtained by making $r-1$ cuts in $X$; after making these cuts we obtain a disc $X^{\prime}$.

We map the boundary of the disc $D_{i j}$ onto that of $D_{i}$ by a covering map of degree $e_{i j}$ which takes vertices to vertices in such a way that a vertex of $\partial D_{i j}$ labeled " $a$ " is mapped to a vertex labeled " $a$ " of $\partial D_{i}$ and so that orientations are preserved. 
Call the map just constructed $f: \partial Y \rightarrow \partial X$; it is unique up to isotopy. We shall show that $f$ can be extended to a covering map $Y \rightarrow X$ of degree $d$.

Let $\Delta$ be the graph in $S^{2}$ obtained as the union of $\partial D_{i j}$, for all $i$ and $j$, and all arcs of the family $\mathbf{C}$. If $\Delta$ is connected, then each component of $S^{2}-\Delta$ is a disc, by the Schoenfliess theorem. In particular each component of $Y^{\prime}$ (the result of cutting $Y$ along $\mathbf{C}$ ) is a disc and the vertex labels of one full circuit, in order, around the boundary of such a disc are

$$
\bar{x}_{r-1} x_{r-1} x_{r-1} \bar{x}_{r-1} \bar{x}_{r-2} x_{r-2} x_{r-2} \bar{x}_{r-2}, \ldots, \bar{x}_{1} x_{1} x_{1} \bar{x}_{1}
$$

(a cyclic word). Since this matches the corresponding cyclic word for $X^{\prime}$ (the result of cutting $X$ ) we can map each disc component of $Y^{\prime}$ homeomorphically onto $X^{\prime}$, extending $f$ along portions of the boundary of $Y$ and $X$, preserving labels of vertices, and such that these maps agree on portions of the boundary which are arcs of $\mathbf{C}$. That is, $f: \partial Y \rightarrow \partial X$ extends to a map $f: Y \rightarrow X$, which is readily seen to be a covering map from the requirements of the preceding sentence. Then we can extend $f$ over $D_{i j}$ to a branched cover $D_{i j} \rightarrow D_{i}$ which is equivalent to the mapping $z \rightarrow z^{e_{i j}}$. In this way $f$ extends to a branched cover $f: S^{2} \rightarrow S^{2}$ whose branch datum is precisely the given datum $\mathbf{D}$. This completes the proof of the sufficiency in Theorem (1.5), provided we assume the graph $\Delta$ is connected. Observe that we have not yet used the hypothesis that $v(\mathbf{D})=2 d-2$.

(2.3) LEMMA. If $v(\mathbf{D})=2 d-2$, then the graph $\Delta$ is connected.

PROOF. Let the connected components of $\Delta$ be the $\Delta_{1}, \Delta_{2}, \ldots, \Delta_{s}$. Each graph $\Delta$ corresponds to an abstract branch datum $\mathbf{D}_{i}$ of degree $d_{i}$ where $\sum_{i=1}^{s} d_{i}=d$. To see this observe that as many positive labels occur on vertices of $\Delta_{i}$ as negative labels. But the number $d_{i}$ of occurrences of $\bar{x}_{j}$ is the same as that of $\bar{x}_{k}$ for any $j$ and $k, 1 \leq j, k \leq r-1$. It follows that $\Delta_{i}$ does indeed arise from a branch datum $\mathrm{D}_{i}$.

By the argument preceding the lemma, since $\Delta_{i}$ is connected, $\mathbf{D}_{i}$ is realized by a branched cover $S^{2} \rightarrow S^{2}$. Hence the Riemann-Hurwitz formula applies and $v\left(\mathbf{D}_{i}\right)=2 d_{i}-2$. Thus $\sum_{i=1}^{s} d_{i}=d$ and $\sum_{i=1}^{s}\left(2 d_{i}-2\right)=2 d-2$. These two equations imply that $s=1$ and hence $\Delta$ is connected, as claimed.

This completes the proof of sufficiency in Theorem (1.5).

We proceed now to establish the necessity of the conditions in Theorem (1.5). Let $f: S^{2} \rightarrow S^{2}$ be a branched cover. An Euler characteristic argument establishes (1.1), so we must only establish (1.6). Let $\left\{P_{1}, P_{2}, \ldots, P_{r}\right\}$ be the image of the set of branch points of $f$. Excise the interiors of pairwise disjoint discs $D_{i}$ centered at $P_{i}$ to obtain

$$
X=S^{2}-\bigcup_{i=1}^{r} \stackrel{\circ}{D}_{i}
$$

Let $Y=f^{-1}(X)$, so $F / Y: Y \rightarrow X$ is an unbranched covering. Mark one point $x_{i}$ on $\partial D_{i}$ for $1 \leq i \leq r-1$ and mark points $\bar{x}_{r-1}, \bar{x}_{r-2}, \ldots, \bar{x}_{1}$ in order on $\partial D_{r}$. Let $\mathbf{C}$ be a cancellation diagram for $x_{1}, x_{2}, \ldots, x_{r-1}, \bar{x}_{r-1} \bar{x}_{r-2} \cdots \bar{x}_{1}$ on $X$. Then $f^{-1}(\mathbf{C})$ is a cancellation diagram for the appropriate collection of words on $Y$. The result (1.6) follows from Theorem 5.2 of [2]. This completes the proof of Theorem (1.5). 
(2.4) REMARK. It can be shown that if $f: M \rightarrow S^{2}$ is a branched covering of degree $d$ where $M$ is a surface of genus $g$ and if the branch datum associated with $f$ is $\mathbf{D}=\left(A_{1}, A_{2}, \ldots, A_{r}\right)$ with $A_{i}=\left\{e_{i 1}, e_{i 2}, \ldots, e_{i r_{i}}\right\}$ then the subset $\prod_{i, j}\left(x_{i}^{e_{i j}}\right)^{\Gamma}$ of $\Gamma$ contains a product of $g$ commutators in $\Gamma$. Here $\Gamma=\left\langle x_{1}, x_{2}, \ldots, x_{r} \mid x_{1} x_{2} \cdots x_{r}\right\rangle$. I do not know whether this condition, together with the Euler characteristic condition (1.1), is sufficient for realizability.

(2.5) REMARK. It is a standard result of complex variables that if an abstract branch datum $\mathrm{D}$ is realized by a branched cover $f: S^{2} \rightarrow S^{2}$ then there is a rational function on $\mathbf{C} P^{1}$ realizing $\mathbf{D}$. Here is a proof of this fact. Pull back the complex structure on $S^{2}$ by the branched cover $f$ to a complex structure on $S^{2}$. Since the complex structure on $S^{2}$ is unique, it follows that, with these structures, $f$ is a rational function on $\mathbf{C} P^{1}$.

(2.6) ExAMPLE. Let $\mathbf{D}=\{[5],[2,2,1],[2,2,1]\}$. Here $d=5$ and $v(\mathbf{D})=8=$ $2 d-2$. Then $\mathrm{D}$ is realizable if

$$
1 \in\left((\bar{x} \bar{y})^{5}\right)^{F}\left(x^{2}\right)^{F}\left(x^{2}\right)^{F}(x)^{F}\left(y^{2}\right)^{F}\left(y^{2}\right)^{F}(y)^{F},
$$

where $F=F(x, y)$ is freely generated by $x$ and $y$, by Theorem (1.5). The diagram below is a cancellation diagram for the words $(\bar{x} \bar{y})^{5}, x^{2}, x^{2}, x, y^{2}, y^{2}, y$ in $F$. It follows that $\mathbf{D}$ is realizable.

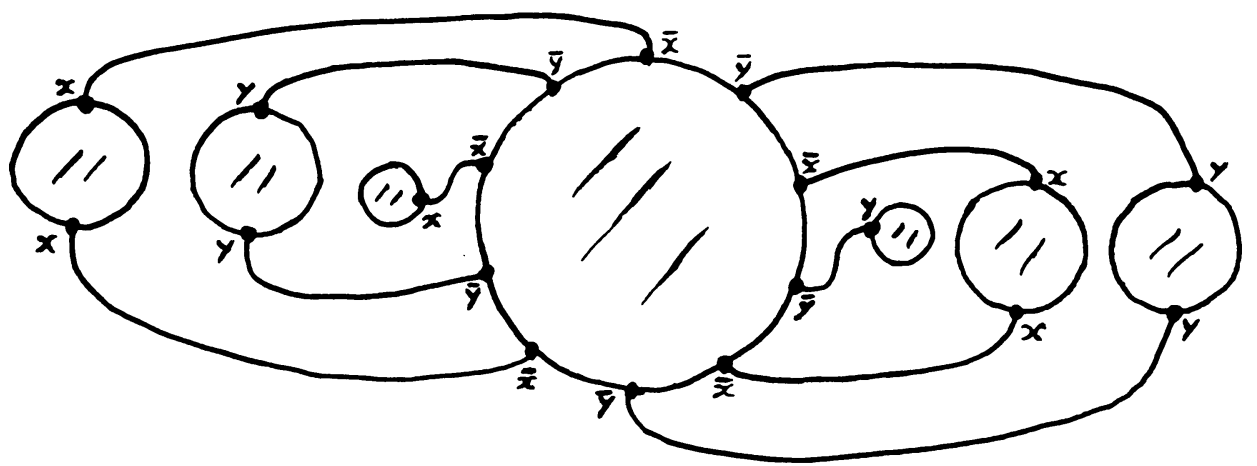

(2.7) REMARK. Let us briefly indicate the connection between Hurwitz' criterion (1.2)-(1.4) and our (1.5) for representability of a branch datum

$$
\mathbf{D}=\left(A_{1}, A_{2}, \ldots, A_{r}\right)
$$

by a branched cover $f: S^{2} \rightarrow S^{2}$, i.e. $\mathbf{D}=\mathbf{D}(f)$. Without loss of generality assume that the image $F=\left\{P_{1}, P_{2}, \ldots, P_{r}\right\}$ of the branch locus of $f$ is the set of $r$ th roots of unity in $\mathbf{C}$, so $P_{j}=e^{2 \pi \sqrt{-1} j / r}, 1 \leq j \leq r$. Take $P_{0}$ to be the origin and let $\xi_{j}$ be a simple closed curve based at $P_{0}$ and winding around $P_{j}$ once in the positive direction, $1 \leq j \leq r$, and such that

$$
\xi_{j} \text { is very close to the ray from } P_{0} \text { to } P_{j},
$$

and

$$
\xi_{j} \text { and } \xi_{k} \text { only meet at } P_{0} \text { for } j \neq k \text {. }
$$


As the model for $S^{2}$ take $\mathbf{C} \cup\{\infty\}$. Let $x_{j}=\left[\xi_{j}\right]$ in $\pi_{1}\left(S^{2}-F, P_{0}\right)$, so $x_{1} x_{2} \cdots x_{r}=1$. We let $\alpha_{j}$ be the monodromy of the circuit $\xi_{j}$ at the generic fiber $f^{-1}\left(P_{0}\right)$ of the covering

$$
f \mid: S^{2}-f^{-1}(F) \rightarrow S^{2}-F .
$$

Then one checks that $\Pi\left(\alpha_{j}\right)=A_{j}$ and that conditions (1.2)-(1.4) are satisfied with $d=\#\left(f^{-1}\left(P_{0}\right)\right)$.

(2.8) REMARK. Hurwitz' solution to the realizability problem (1.2)-(1.4) is effective. The referee inquired whether our condition (1.6) is effective. In fact this is the case, as follows from methods developed in R. Z. Goldstein and E. C. Turner (Math Z. 165 (1979), 1-10), applied to a finite collection of words in a free group.

\section{REFERENCES}

1. A. L. Edmonds, R. S. Kulkarni, and R. E. Stong, Realizability of branched coverings of surfaces, Trans. Amer. Math. Soc. 282 (1984), 773-790.

2. S. M. Gersten, Products of conjugacy classes in a free group: a counterexample, Math. Z. 192 (1986), 167-181.

3. A. Hurwitz, Ueber Riemann'sche Flachen mit gegebener Verzweigungspunkten, Math. Ann. 39 (1891), 1-61.

Department of Matihematics, University of Utah, Salt lake City, Utah 84112 